\title{
A BANACH ALGEBRA CRITERION FOR TAUBERIAN THEOREMS
}

\author{
I. DAVID BERG ${ }^{1}$
}

We acknowledge valuable discussions with Dr. J. Lindenstrauss of Yale University.

An infinite matrix $A$ is called conservative if for each convergent sequence $x$ the transformed sequence $A x$ is convergent. It is a classical theorem that a necessary and sufficient condition that a matrix $A$ be conservative is that:

(1) $\|A\|=\sup _{i} \sum_{j=1}^{\infty}\left|a_{i j}\right|<\infty$,

(2) $\lim _{i \rightarrow \infty} \sum_{j=1}^{\infty} a_{i j}$ exists,

(3) $\lim _{j \rightarrow \infty} a_{i j}$ exists for each $i$.

It is well known that the set of conservative matrices forms a Banach algebra; we will call this Banach algebra $\Gamma$.

Throughout this paper "matrix" will mean "conservative matrix" unless otherwise stated.

Many criteria for a matrix to sum no bounded divergent sequence are known; cf. Copping [1], Wilansky and Zeller [2], [4].

The purpose of this paper is to show that if $A$ is not a left divisor of zero (abbreviated 1.z.d.) in $\Gamma$ then a necessary and sufficient condition that $A$ sum some bounded divergent sequence is that $A$ be a left topological divisor of zero (abbreviated 1.t.z.d.) in $\Gamma$ (i.e. for $\epsilon>0$ there exists $B \in \Gamma$ such that $\|B\|=1$ and $\|A B\|<\epsilon)$. Equivalently, a necessary and sufficient condition that $A, A$ not a l.z.d., sum a bounded divergent sequence is the following:

For $\epsilon>0$ there exists a convergent sequence, $x$, such that $\|x\|$ $=\sup _{n}|x(n)|=1$ and $\|A x\|<\epsilon$.

Copping [1] proved a theorem conjectured by Wilansky and Zeller [3] which we paraphrase as follows:

A triangular $\left(a_{n, k}=0\right.$ for $\left.k>n\right)$ conservative matrix not a l.z.d. sums $a$ bounded divergent sequence if it is on the boundary of the maximal group.

We shall show that this theorem is true even if "triangular" is omitted. As Copping [1] shows, the converse is false. Hence there are 1.t.z.d.'s in the Banach algebra, $\Delta$, of conservative triangular matrices not on the boundary of the maximal group. However the structure of the set of 1.t.z.d.'s in both $\Delta$ and $\Gamma$ still eludes us.

Received by the editors January 28, 1963 and, in revised form, March 14, 1963.

1 This research was supported in part by N. S. F. grant G-19041. 
We first note that $A$ is a l.t.z.d. iff for $\epsilon>0$ there exists a convergent sequence $x$ such that $\|x\| \geqq 1$ and $\|A x\|<\epsilon$. Indeed, if $A$ is a l.t.z.d., choose $B$ such that $\|B\|>1$ and $\|A B\|<\epsilon$. If we let $b_{i}$ represent the $i$ th column of $B$, we see we may choose scalars $a_{i}$ of modulus 1 and $n$ such that $x=\sum_{i=1}^{n} a_{i} b_{i}$ is the desired sequence. The converse is clear.

It is also obvious that $A$ is a l.z.d. iff there exists a convergent sequence $x \neq 0$ such that $A x=0$. Observe that if $x$ is a convergent sequence the matrix whose first column is $x$ and all of whose other columns are 0 is a trivial conservative matrix.

We observe that the requirement that $A$ is not a l.z.d. is a trifle weaker than the requirement that $A$ defines a $U$-method [1] or the requirement that $A$ is reversible [2]. For if $A$ is reversible, $A$ cannot annihilate any sequence; if $A$ defines a $U$-method, $A$ cannot annihilate any bounded sequence. But if $A$ is not a l.z.d. we require only that $A$ cannot annihilate any convergent sequence.

We pause here to introduce some convenient notation.

Let $x$ be a sequence. Define the sequence $T_{m, n} x$ by

$$
\begin{aligned}
T_{m, n} x(p) & =x(p) & & \text { for } m \leqq p \leqq n, \\
& =0 & & \text { otherwise. }
\end{aligned}
$$

Define the sequence $T_{m} x$ by

$$
\begin{aligned}
T_{m} x(p) & =x(p) & & \text { for } p \geqq m, \\
& =0 & & \text { otherwise. }
\end{aligned}
$$

We must now introduce some computational lemmas.

Lemma 1. Let $A$ be a l.t.z.d. not a l.z.d. Then for $\epsilon>0$ and integer $n>0$ there exists a convergent sequence $x$ of norm 1 such that $T_{n} x=x$ and $\|A x\|<\epsilon$.

Proof. There exists convergent $x_{i}$ such that $\left\|x_{i}\right\|=1$ and $\left\|A x_{i}\right\|$ $<i^{-1}$. Suppose there exists $n$ such that for some $\lambda>0$ if $T_{n} x=x$ then $\|A x\| \geqq \lambda\|x\|$. Let $a_{i}=T_{1, n-1} x_{i}, b_{i}=T_{n} x_{i}$. Then $x_{i}=a_{i}+b_{i}$.

Select a convergent subsequence of the $a_{i}$, call it $a_{i_{k}}$. Let $\lim _{k} a_{i_{k}}=a$. Then $\lim _{k \rightarrow \infty} A\left(a_{i_{k}}+b_{i_{k}}\right)=A a+\lim _{k \rightarrow \infty} A b_{i_{k}}=0$.

Hence $\lim _{k \rightarrow \infty} A b_{i_{k}}$ exists. Since for any convergent $x$

$$
\left\|A T_{n} x\right\| \geqq \lambda\left\|T_{n} x\right\|,
$$

we see that $\lim _{k \rightarrow \infty} b_{i_{k}}$ exists; call it $b$. Hence $A(a+b)=0$.

Since $a+b$ is convergent and $(a+b) \neq 0$, we see that $A$ is a l.z.d. This completes the proof. 
Lemmas 2 and 3 are essentially well known.

Lemma 2. Let Ay converge for some bounded sequence $y$. Then

(1) $A T_{n} y$ is convergent for each fixed $n$; i.e. $\lim _{p \rightarrow \infty}\left(A T_{n} y\right)(p)$ exists for each $n$,

(2) $\lim _{p}\left(A T_{n} y\right)(p)$ is a convergent sequence in $n$,

(3) $\lim _{n}\left(A T_{n} y\right)(p)=0$ for each fixed $p$.

Proof. (1) is immediate. To see (2), observe that

$$
\left|\lim _{D}\left(A T_{n} y\right)(p)-\lim _{D}\left(A T_{m} y\right)(p)\right| \leqq\left(\sum_{i=m}^{n}\left|\lim _{j} a_{i j}\right|\right)\|y\| .
$$

Since $A$ is bounded, $\sum_{i=1}^{\infty}\left|\lim _{j} a_{i j}\right|<\infty$.

The boundedness of $A$ yields (3), since $\left|\left(A T_{n} y\right)(p)\right|$ $\leqq\left(\sum_{j=n}^{\infty}\left|a_{p j}\right|\right)\|y\|$. This completes the proof.

Lemma 3. Let Ay converge for some bounded divergent sequence $y$. Then for $\epsilon>0$, there exists a bounded divergent sequence $z$ such that:

(1) $\lim _{p, q} \sup |z(p)-z(q)|>1$,

(2) $\left|\lim A T_{n} z\right|<\epsilon$ for all $n$.

Proof. We first consider the case where $A$ is coregular: i.e. $\chi(A)$ $\equiv\left(\lim _{i} \sum_{j=1}^{\infty} a_{i j}-\sum_{j=1}^{\infty} \lim _{i} a_{i j}\right) \neq 0$. We may assume

$$
\lim _{p, q} \sup _{p}|y(p)-y(q)|>1 \text {. }
$$

For $\epsilon>0$, by Lemma 2 we may choose a convergent sequence $x$ and an integer $N$ so that for $m, n \geqq N$ :

$$
\left|\lim _{p}\left(A T_{n} x\right)(p)-\lim _{p}\left(A T_{m} y\right)(p)\right|<\epsilon .
$$

Then $z=T_{N} x-T_{N} y$ is our desired sequence.

We now consider the case where $A$ is conull, i.e. $\chi(A)=0$. In this case, letting $x=(1,1,1, \cdots)$ we see that $\lim _{n} \lim _{p}\left(A T_{n} x\right)(p)=0$. For appropriate scalars $a_{j}$, where $\left|a_{j}\right|=j^{-1}$, and appropriate integers $n_{j}$ we see that we may construct a sequence $z$, defined coordinatewise by

$$
z=\sum_{j=1}^{\infty} a_{j} T_{n_{j}} x,
$$

which will be the desired bounded divergent sequence. This is a standard sort of gliding hump argument; the details are similar to those of Theorem 2. This completes the proof. We come now to our first result. 
Theorem 1. Let $A$ sum a bounded divergent sequence. Then $A$ is a l.t.z.d.

Proof. We may assume $\|A\|=1$. By Lemma 3 , for $\epsilon>0$ we may choose a bounded divergent sequence, $z$, such that

(1) $\left|\lim A T_{n} z\right|<\epsilon / 4$ for all $n$,

(2) $|\lim \sup z|>1$.

We will now build out of sums of $T_{n} z$ for appropriate $n$ 's a finite sequence $x$ such that $\|x\| \geqq 1$ but $\|A x\|<\epsilon$. Pick integer $p$ so that $1 / p<\epsilon / 4\|z\|$ and consider

$$
x=\frac{1}{p}\left(T_{n_{1}} z+T_{n_{2}} z+\cdots+T_{n_{p}} z-T_{n_{p+1}} z-\cdots-T_{n_{2 p}} z\right),
$$

where $n_{i}$ is chosen as follows:

$n_{1}=1$. Choose $n_{i}, i>1$, so that

$$
\left|\left(A T_{n_{j}} z\right)(q)\right|<\epsilon / 4 \quad \text { for } j<i \text { and } q \geqq n_{i} \text {, }
$$

and

$$
\left|\left(A T_{n_{i}} z\right)(q)\right|<\epsilon / 4 \quad \text { for } q \leqq n_{i-1} \text {. }
$$

(That is, we choose $n_{i}$ so large that all early terms in $A T_{n_{i}} z$ are small and so that for each previous $n_{j}$ the terms in $A T_{n_{j}} z$ are small by the $n_{i}$ th term.)

Choose $n_{p+1}$ so that in addition to the preceding requirements, $|z(q)|>1$ for some $q$ such that $n_{p}<q<n_{p+1}$. We now see that $\|x\|>1$ and that for $q \geqq n_{2 p}, x(q)=0$. However

$$
\|A x\|<(1 / p)(2 p(\epsilon / 4)+2\|z\|)<\epsilon .
$$

By our remarks preceding Lemma 1 we see that $A$ is a l.t.z.d. This completes the proof.

Theorem 2. Let $A$ be a l.t.z.d. not a l.z.d. Then $A$ sums a bounded divergent sequence.

Proof. We assume $\|A\|=1$. Choose a set $z_{n}$ of convergent sequences such that

(1) $\left\|z_{n}\right\|=1$ for each $n$,

(2) $\left\|A z_{n}\right\|<1 / 2^{n}$,

(3) $z_{n}(p)=0$ for $p \leqq k_{n}$, where $k_{n}$ is chosen so that $k_{n}>k_{n-1}$ and $\left|z_{n-1}(p)-\lim z_{n-1}\right|<1 / 2^{n}$ for $p \geqq k_{n}, k_{1}$ arbitrary.

This is possible by Lemma 1 .

Now choose a set of scalars $a_{n}$ of modulus 1 so that the coordinatewise limit of $\sum_{n=1}^{\infty} a_{n} z_{n}$ is a bounded divergent sequence, call it $x$. and so that 


$$
\left\|\sum_{n=1}^{p} a_{n} z_{n}\right\|<M \quad \text { for all } p .
$$

Now by Lemma 2 we observe that for fixed $p$ and $\epsilon>0$ we have

$$
\left|\left(A x-\sum_{n=1}^{q} A a_{n} z_{n}\right)(p)\right|<\epsilon M
$$

for large enough $q$.

Hence $A x=\sum_{n=1}^{\infty} a_{n} A z_{n}$ which is the uniform limit of convergent sequences. This completes the proof.

At this point we observe that the proofs (especially of Theorem 1) could have been shortened by use of Theorem 1, p. 502 of Wilansky and Zeller [4]. However, we prefer to keep the proofs self-contained.

We now have the following

Corollary. Let $A$ be on the boundary of the maximal group of $\Gamma$ (i.e. $A$ is not invertible in $\Gamma$, but is in the closure of the group of invertible elements of $\Gamma$ ). Let $A$ not be a l.z.d. Then $A$ sums a bounded divergent sequence.

Recalling that any element on the boundary of the maximal group in any Banach algebra is a 2-sided topological divisor of zero, this corollary is immediate.

\section{REFERENCES}

1. J. Copping, Mercerian theorems and inverse transformations, Studia Math. 21 (1962), 177-194.

2. A. Wilansky and $\mathrm{K}$. Zeller, The inverse matrix in summability: reversible methods, J. London Math. Soc. 32 (1957), 397-408.

3. - Banach algebra and summability, Illinois J. Math. 2 (1958), 378-385.

4. - Summation of bounded divergent sequences, topological methods, Trans. Amer. Math. Soc. 78 (1955), 501-509.

YALE UNIVERSITY 